\title{
USO DO ESCORE PROGNÓSTICO APACHE II E ATN-ISS EM INSUFICIÊNCIA RENAL AGUDA TRATADA DENTRO E FORA DA UNIDADE DE TERAPIA
} INTENSIVA

\author{
*Natália Maria da Silva Fernandes ${ }^{1}$, Patrícia dos Santos Pinto², Thiago Bento de Paiva Lacet³ , Dominique Fonseca Rodrigues ${ }^{3}$, Marcus Gomes Bastos ${ }^{4}$, \\ Sérgio Reinaldo Stella ${ }^{5}$, Miguel Cendoroglo Neto ${ }^{5}$ \\ Trabalho realizado na Universidade Federal de Juiz de Fora - UFJF, Minas Gerais, MG, e Universidade Federal do Estado de São Paulo - UNIFESP, S. Paulo, SP
}

*Correspondência:

Rua Jamil Altaff, nº 132 -

Vale do Ipê

Juiz de Fora - MG

CEP: $36035-380$
RESUMO

INTRODuçăo. A insuficiência renal aguda (IRA) mantém alta prevalência, morbidade e mortalidade. OBJetivo. Comparar o uso do escore prognóstico APACHE II com o ATN-ISS e determinar se o APACHE II pode ser utilizado para pacientes com IRA, fora da UTI.

MÉTodos. Coorte prospectiva, 205 pacientes com IRA. Analisamos dados demográficos, condições pré-existentes, falência de órgãos e características da IRA. Os escores prognósticos foram realizados no dia da avaliação do nefrologista.

Resultados. A média de idade foi $52 \pm 18$ anos, $50 \%$ eram do sexo masculino, $69 \%$ eram brancos, $45 \%$ foram tratados em UTI e $55 \%$ em outras unidades. A mortalidade no grupo UTI foi $85 \%$ e no grupo não-UTI foi $18 \%$. Os fatores que se correlacionaram com maior mortalidade foram mais prevalentes na UTI: idade, sexo masculino, IRA hospitalar, falência de órgãos, sepse, IRA séptica, oligúria e necessidade dialítica. No contexto geral, os marcadores prognósticos foram os mesmos para os grupos UTI e não-UTI. O APACHE II obteve discriminação similar nos grupos UTI e não-UTI e sua calibração foi melhor no grupo não-UTI. O ATN-ISS obteve boa discriminação tanto no grupo UTI quanto não-UTI, porém, com relação à calibração houve discreta superestimação da mortalidade no grupo não-UTI. O ATN-ISS apresentou melhor capacidade de discriminação do que o APACHE II nos grupos UTI e não-UTI.

ConcLusÃo. Concluímos que os escores APACHE II e ATN-ISS podem ser utilizados para a estratificação de risco em pacientes com IRA tratados fora da UTI em nosso meio.

UNITERMOS: APACHE II. ATN-ISS. Insuficiência renal aguda. Unidade de terapia intensiva.

\section{INTRODUÇÃO}

A determinação do prognóstico de um paciente tem importância em nível de planejamento do tratamento, custos e prevenção de doenças. Além disso, a medicina está avançando e, constantemente, oferece novas alternativas que merecem avaliação. No caso da insuficiência renal aguda (IRA) não podemos afirmar se certa atitude terapêutica (tipo de diálise, nutrição, etc) é melhor ou pior do que outra se as duas populações analisadas não forem comparáveis. Para resolver este tipo de problema é preciso mensurar a gravidade da doença destas populações utilizando escores prognósticos ${ }^{1}$.

A IRA apresenta elevada morbimortalidade e elevado custo. Complica aproximadamente $5 \%$ das admissões hospitalares ${ }^{2} \mathrm{e}$ até $30 \%$ das admissões em $\mathrm{UTI}^{3}$. Esses números são maiores do que os registrados em pacientes fora da UTI ( $2 \%$ a $5 \%$ ) ou na comunidade $(1 \%)^{4,5}$.

Se examinarmos a taxa de mortalidade nas últimas cinco décadas em pacientes portadores de IRA, observamos que ela permaneceu elevada, apesar dos grandes avanços tecnológicos, terapêuticos e preventivos ocorridos no mesmo período. Houve uma mudança demográfica no perfil do paciente que desenvolve IRA, piorando o seu prognóstico e anulando os efeitos positivos dos avanços tecnológicos nos cuidados com pacientes graves ${ }^{6,7}$.

O escore mais citado na literatura foi criado por Knaus et al. em 1981 e denominado APACHE. Este escore foi revisto em $1985^{8}$ - APACHE II, em 1991 - APACHE III e, em 2005, APACHE IV ${ }^{10}$.

Os resultados com a utilização do APACHE II e de outros escores inespecíficos em pacientes com IRA não têm sido

1. Médica nefrologista da Universidade Federal de Juiz de Fora - UFJF, Juiz de Fora, MG

2. Médica residente em Nefrologia na Universidade Federal de Juiz de Fora - UFJF, Juiz de Fora, MG

3. Acadêmico de medicina da Universidade Federal de Juiz de Fora - UFJF, Juiz de Fora, MG

4. Professor doutor da Universidade Federal de Juiz de Fora - UFJF, Juiz de Fora, MG

5. Professor doutor da Universidade Federal de São Paulo - UNIFESP, São Paulo, SP 
animadores $^{6}$. A insatisfação com o desempenho do APACHE II, e outros escores inespecíficos em pacientes com IRA, levou à criação de escores específicos para este grupo de pacientes por vários autores.

Vários índices prognósticos específicos para IRA foram estudados em subpopulações de pacientes portadores de IRA, a maioria de difícil reprodutibilidade e estudados retrospectivamente. Liaño $F^{11,12}$, em 1993, para analisar a influência de fatores de risco no prognóstico da necrose tubular aguda (NTA), realizou um estudo com um protocolo contendo dados demográficos, causas de NTA, diurese, necessidade de diálise e condições clínicas.

A maioria dos trabalhos analisou pacientes em UTI. A despeito da prevalência de IRA fora da UTI ser significante, poucos estudos avaliaram a validade dos índices prognósticos nesta população7,13,14,15. Alguns estudos, citados previamente, utilizaram escores prognósticos específicos para IRA nesta população, porém o fizeram em subpopulações (IRA póscirúrgica, com necessidade dialítica, etc), dificultando sua reprodutibilidade ${ }^{13-18}$.

Assim, considerando que a IRA apresenta elevado custo e alta morbimortalidade; que há poucos estudos epidemiológicos sobre IRA tratada fora da UTI; que o uso do APACHE II em IRA é controverso e sua utilização não foi validada em pacientes com IRA fora da UTI; que o ATN-ISS é um escore específico, de fácil reprodutibilidade; decidimos realizar um estudo de coorte prospectivo para avaliar as características da IRA na UTI comparando-a com a IRA fora da UTI (não-UTI); estabelecer se o APACHE II pode ser utilizado na população com IRA não-UTI e comparar o APACHE II com o ATN-ISS.

\section{MÉTodos}

Realizamos um estudo de coorte prospectivo no Hospital São Paulo, um hospital terciário, de ensino, situado em São Paulo - Brasil, no período de $1^{\circ}$ de fevereiro de 1997 a 30 de novembro de 1997. O estudo foi aprovado pelo Comitê de Ética e Pesquisa Clínica.

Critérios de inclusão: Foram incluídos todos os pacientes acima de 12 anos de idade tratados ou não na UTI, com diagnóstico de IRA, que foram avaliados e acompanhados pelo Serviço de Interconsulta de Nefrologia.

Critérios de exclusão: Foram excluídos pacientes com menos de 12 anos de idade, com IRA pré-renal, transplantados renais, portadores de insuficiência renal crônica definida por creatinina acima de $2 \mathrm{mg} / \mathrm{dL}$ à admissão, dialisados por intoxicação exógena e aqueles transferidos de hospital durante o tratamento.

População estudada: Foram acompanhados 205 pacientes no período de estudo, sendo nove excluídos por terem sido transferidos de hospital. Assim, a população final estudada foi de 196 pacientes. Dezenove pacientes (10\% do total de pacientes estudados) foram tratados como pacientes de terapia intensiva em unidades de cuidados intermediários ou semi-intensivas: pronto-socorro, enfermaria de doenças infecciosas e parasitárias, enfermaria de gastroenterologia clínica, enfermaria de clínica médica. Esses 19 pacientes não diferiram dos pacientes tratados em UTI com relação a dados demográficos, condições pré-existentes e média de escores prognósticos. Assim, eles foram analisados em conjunto com os pacientes tratados nas
UTIs. Os pacientes foram acompanhados até a alta ou óbito intra-hospitalar.

\section{MÉTodos}

Os pacientes com IRA foram localizados através da solicitação de avaliação pelo médico assistente. Os critérios utilizados para definição e categorias etiológicas da IRA foram os critérios publicados por Liaño et al., $1993^{12}$.

Liaño $F^{11,12}$, para analisar a influência de fatores de risco no prognóstico da NTA, realizou um estudo com um protocolo contendo dados demográficos, causas de NTA, diurese, necessidade de diálise e condições clínicas. A equação obtida foi:

ATN-ISS $=0,032$ (idade em décadas) - 0,086 (sexo) 0,109 (nefrotóxica) 0,109 (oligúria) 0,116 (hipotensão) 0,122 (icterícia) 0,150 (coma) - 0,154 (consciente) 0,182 (ventilação mecânica) 0,21.

ATN-ISS: escore de gravidade individual de necrose tubular aguda. Variáveis definidas como e com os seguintes valores: Hipotensão: PAS $<100 \mathrm{mmHg}$ por mais de 10 horas com ou sem vasopressores -1 . Oligúria - diurese $<400 \mathrm{ml} /$ dia -1 . Icterícia: bilirrubinas $>2 \mathrm{mg} / \mathrm{dl}$ - 1 . Coma -1 . Consciência normal - 1 . Ventilação mecânica - 1. Sexo masculino - 1. Idade em décadas - 1 a 11. Ausência de qualquer destas características - 0 .

Estes dados devem ser obtidos no momento da avaliação do nefrologista ou na internação do paciente se a mesma deu-se por NTA. O ponto de corte obtido foi 0,9 , ou seja, acima deste não houve sobrevida nas quatro fases (1). Insuficiências orgânicas foram definidas segundo Knaus et al., 1985'19. O escore ATN-ISS 12 foi calculado no dia da avaliação do nefrologista, assim como o escore APACHE $\|^{8}$ e o risco de óbito calculado. Os escores foram realizados somente pela pesquisadora. Os desfechos estudados foram: óbito no hospital, necessidade de diálise, recuperação de função renal.

\section{AnÁlise estatística}

Os dados foram expressos como média, desvio padrão, percentagem ou número absoluto. Para a análise estatística dos resultados foram aplicados os seguintes testes:

Análise univariada: 1 - Teste Qui quadrado: para análise de tabelas de contingência. 2 - Teste exato de Fisher: quando não foi possível a utilização do teste Qui quadrado pelo tamanho da amostra. 3 - Teste T de Student: para a comparação de duas amostras não pareadas de variáveis quantitativas contínuas.

Análise multivariada: 1- Regressão logística "Stepwise": as variáveis consideradas estatisticamente significantes na análise univariada foram incluídas em modelos para análise multivariada.

Análise dos escores: 1- Análise de discriminação: a capacidade discriminatória dos escores em distinguir sobreviventes de não sobreviventes, foi avaliada pela curva receiver operator characteristic (ROC) com sua respectiva área sob a curva (AUC). Essa curva mostra a relação entre a sensibilidade (identificação correta dos não sobreviventes) e um menos especificidade (identificação incorreta daqueles que sobrevivem). A técnica de discriminação nos propicia a seguinte análise: se todos os possíveis pares de pacientes em que um sobreviveu e outro morreu forem identificados, a área sob a curva (AUC) pode ser 


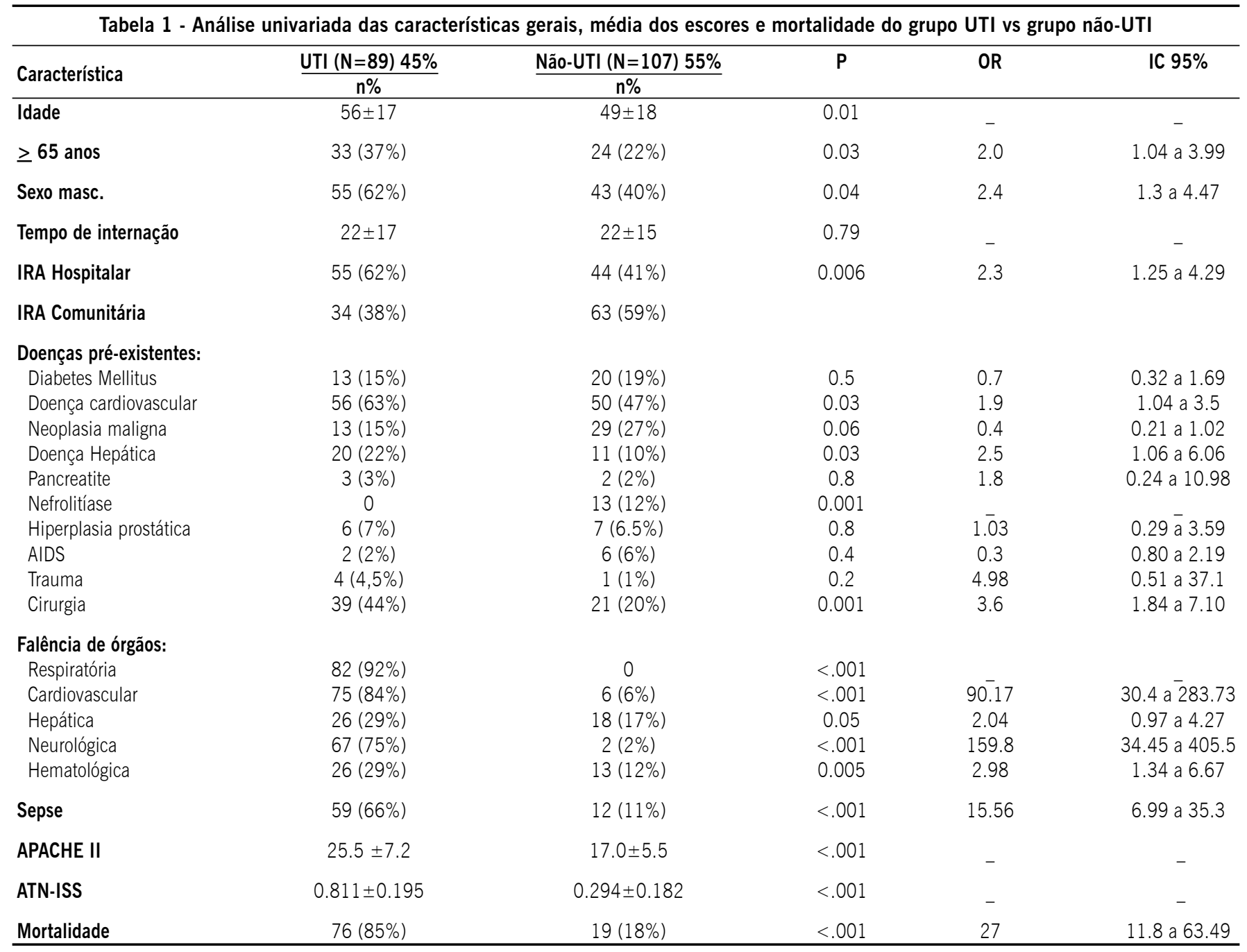

interpretada como a proporção de pares em que o modelo pode corretamente identificar o paciente que morreu. Se a área sob a curva é 0,5 , então o modelo não tem poder discriminatório e, se a área é 1,0, o modelo discrimina perfeitamente. Em outras palavras, a discriminação diz respeito à capacidade do escore acertar o desfecho (ex: morte ou sobrevida) de cada indivíduo. As comparações da AUC obtidas para o risco-APACHE II e ATN-ISS, ou escores obtidos para o grupo UTI e grupo não-UTI foram feitas de acordo com a técnica descrita por Hanley ${ }^{20,21}$. 2- Calibração: a calibração refere-se ao grau de correspondência entre a probabilidade de morte predita e observada. Em outras palavras, a calibração diz respeito ao quanto o escore tende a subestimar ou superestimar a ocorrência de um desfecho em cada estrato da população estudada, independentemente da sua capacidade de acerto. Para avaliá-la, separamos os pacientes em quintiles de risco para cada um dos escores nos grupos UTI e não-UTI. Em cada quintil de risco a mortalidade predita foi comparada com a mortalidade observada. Avaliamos a adequação do ajuste das curvas pelo teste de Goodness of Fit.
Todas as diferenças observadas foram consideradas significantes se $p \leq 0,05$. Para a realização dos testes, foi utilizado o software estatístico True Epistat, (Tracy Gustaffson, Richardson, Texas, 1994).

\section{Resultados}

Comparação entre a população UTI e NUTI:

No período de $1^{\circ}$ de fevereiro a 30 de novembro de 1997 foram internados 19.497 pacientes no Hospital São Paulo (excluindo-se pediatria e UTI neonatal). Destes, 205 (1,05\%) apresentaram IRA. Nove destes foram transferidos de hospital. Assim, foi incluído no estudo um total de 196 pacientes, divididos em dois grupos para análise: pacientes que foram tratados em UTI (grupo UTI) e pacientes que não foram tratados em UTI (grupo não-UTI). O tempo médio de internação dos pacientes que apresentaram IRA foi maior do que a média geral do hospital (22,3 dias e 7,6 dias, $p=0.001$ ). A mortalidade dos pacientes que apresentaram IRA também foi maior do que a mortalidade geral do hospital (48\% e 6\%, $p=0.0001$ ). O tempo médio de IRA 


\begin{tabular}{|c|c|c|c|c|c|}
\hline Séptica & $56(63 \%)$ & $10(9 \%)$ & $<.001$ & 16.46 & 7.12 a 38.97 \\
\hline Cirúrgica & $7(8 \%)$ & $10(9 \%)$ & 0.9 & 0.82 & 0.26 a 2.49 \\
\hline Clínica & $22(25 \%)$ & $62(58 \%)$ & $<.001$ & 0.2 & 0.1 a 0.39 \\
\hline Oligúria & $55(62 \%)$ & $35(33 \%)$ & 0.001 & 2.5 & $1.4 \bar{a} 4.7$ \\
\hline Necessidade de diálise & $43(48 \%)$ & $25(23 \%)$ & 0.001 & 3.0 & 1.59 a 5.92 \\
\hline \multicolumn{6}{|l|}{ Tipo de diálise: } \\
\hline $\mathrm{HDI}$ & $9(10 \%)$ & $20(19 \%)$ & 0.13 & 0.48 & 0.19 a 1.21 \\
\hline CVVHD & $27(30 \%)$ & $1(1 \%)$ & $<.001$ & 46.16 & 6.43 a 166.97 \\
\hline Recuperação de função renal & $26(29 \%)$ & $63(59 \%)$ & 0.00006 & 0.28 & 0.15 a 0.54 \\
\hline
\end{tabular}

foi de 17 dias, e o tempo médio até a avaliação de nefrologista por dois dias.

$\mathrm{Na}$ Tabela 1, verificamos as características gerais, média dos escores e mortalidade nos grupos UTI e não-UTI. Nota-se que os pacientes do grupo UTI são mais idosos que os do grupo não-UTI, a maioria é do sexo masculino, com IRA de origem hospitalar, apresentam mais doença cardiovascular, neoplasia maligna, hepatopatia e cirurgia.

Com relação aos escores prognósticos, o grupo UTI apresentou maior pontuação tanto no APACHE II quanto no ATN-ISS, demonstrando a maior gravidade dos pacientes incluídos nesse grupo. A mortalidade global foi de $48 \%$, sendo $85 \%$ no grupo UTI e $18 \%$ no grupo não-UTI.

Na Tabela 2, analisamos os dados coletados no dia da avaliação do nefrologista, através de análise univariada. Foram avaliadas as causas da IRA, nível de creatinina, necessidade dialítica, tipo de diálise e recuperação de função renal nos dois grupos. No grupo UTI, a maioria dos pacientes apresentou IRA devido à sepse (63\%), enquanto no grupo não-UTI, IRA clínica foi a maior causa (58\%).

Casos de IRA pós-renal não foram observados em pacientes do grupo UTI, mas esteve presente em $15 \%$ dos pacientes do outro grupo. Quanto à frequência de nefrotoxicidade e IRA pós-cirúrgica, não houve diferença significativa entre os grupos. Sessenta e dois por cento dos pacientes do grupo UTI tornaram-se oligúricos e $48 \%$ necessitaram de diálise, enquanto no grupo não-UTI essas taxas foram $33 \%$ e $23 \%$, respectivamente. Não houve diferença entre as modalidades de diálise utilizadas nos dois grupos, com exceção da CVVHD, que foi mais utilizada no grupo UTI. O grupo não-UTI apresentou maior taxa de recuperação da função renal (59\%) quando comparada ao grupo UTI (29\%).

Realizamos também análise univariada, comparando sobreviventes e não sobreviventes. Observamos que, dentre os fatores associados à mortalidade, as variáveis idade $(p=0,003, I C=1$ a 1,04$)$, sexo masculino $(p=0,03, I C=1,48$ a 3,11), falência respiratória $(p=0,02, I C=1,8$ a 25,8$)$, falência cardiovascular
( $p=0,007, I C=1,64$ a 23,2), falência neurológica $(p=0,06$, $\mathrm{IC}=1,65$ a 27,4$)$, falência hepática $(p=0,005, I C=1,55$ a $13,47)$, choque séptico ( $p<0,00000, I C=16,2$ a 211 ) e oligúria $(p=0,04, I C=1$ a 6,3$)$ foram associados à maior mortalidade $e$ mantiveram-se após análise multivariada. Estas mesmas características foram mais prevalentes em pacientes com IRA na UTI.

\section{ANÁLISE DOS ESCORES}

\section{Discriminação}

No Gráfico 1- Curva A observamos a área sob a curva (AUC) do ATN-ISS do grupo UTI e no Gráfico 1 - Curva B a AUC para o grupo não-UTI. As AUC foram respectivamente de 0,94 e 0,93. O Gráfico 1 - Curva C mostra a AUC para o risco-APACHE II para o grupo UTI e o Gráfico 1- Curva D para os pacientes do grupo não-UTI, com uma AUC, respectivamente, de 0,78 e 0,66.

Não houve diferença estatisticamente significante entre as AUC dos escores ATN-ISS e risco-APACHE II, comparando-se o grupo UTI e grupo não-UTI (ATN-ISS: AUC UTI=0,94, AUC $\mathrm{NUTI}=0,93 ; p=0,51$; risco-APACHE II: $A \cup C \cup T I=0,78 ; A \cup C$ $\mathrm{NUTI}=0,66 ; p=0,16)$. Quando comparamos a AUC do ATN-ISS e do risco-APACHE II em cada grupo, observamos que o ATN-ISS apresentou melhor desempenho do que o risco-APACHE II no grupo UTI (ATN-ISS AUC=0,94, risco-APACHE $\| A \cup C=0,78$; $p=0,01$ ). O mesmo pode ser observado no grupo não-UTI (ATNISS AUC $=0,93$, risco-APACHE $\|=0,66 ; p<0,001$ ).

\section{Calibração}

Separamos para cada grupo os escores ATN-ISS e o riscoAPACHE II em quintiles de risco e avaliamos a adequação do ajuste da mortalidade predita e mortalidade observada. Na comparação entre a mortalidade predita e observada para o ATN-ISS no grupo UTI não houve diferença estatística $(p=0,9)$. Comparando o ATN-ISS no grupo não-UTI houve diferença estatística entre a mortalidade predita e observada e, neste grupo, este escore superestimou a mortalidade $(p=0,01)$. A mortalidade 


\section{Gráfico 1 - Curva ROC (receiver operator curve) para ATN-ISS e APACHE II em pacientes com IRA tratados em UTI e fora da UTI}

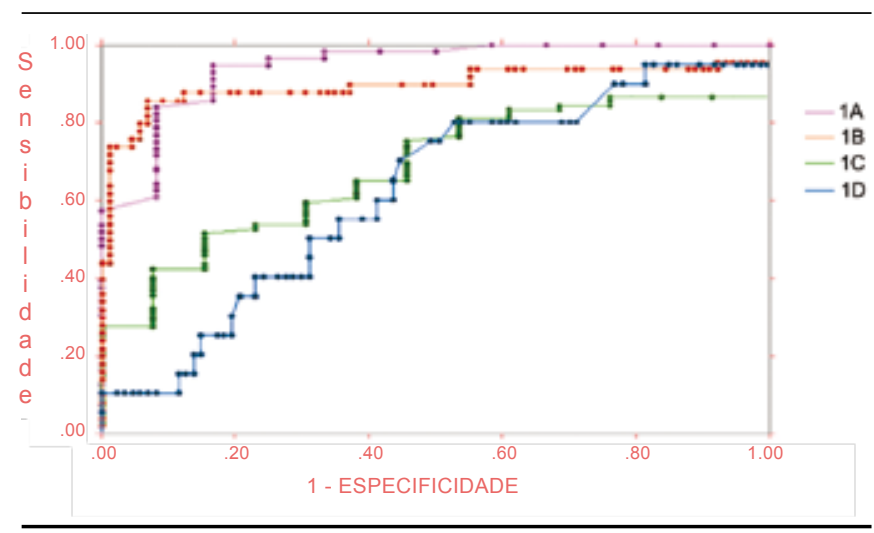

Curva 1A para ATN-ISS em pacientes com IRA tratados em UTI (área sobre a curva $=0,94$; erro padrão=0,035; $p<0,000001$ ).

Curva 1B para ATN-ISS para pacientes com IRA tratados fora da UTI (área sobre a curva $=0,93$; erro padrão $=0,035 ; p<0,000001$ ).

Curva $1 \mathrm{C}$ para risco-APACHE II em pacientes com IRA tratados em UTI (área sobre a curva $=0,78$; erro padrão $=0,0662 ; p=0,000008$ ).

Curva 1D para risco-APACHE II em pacientes com IRA tratados fora da UTI (área sobre a curva $=0,66$; erro padrão $=0,0627 ; p=0,006$ ).

predita e observada do risco-APACHE II na população tratada na UTI mostrou que houve diferença estatística entre as duas curvas e que, neste grupo, o escore subestimou a mortalidade $(p<0,000001)$. A mortalidade predita e observada do riscoAPACHE II no grupo não-UTI mostra uma boa concordância entre as duas curvas, não tendo ocorrido diferença estatística $(p=0,47)$.

\section{Dıscussão}

\section{Epidemiologia da IRA}

A falta de uma definição uniforme para IRA é um dos principais impedimentos para pesquisas nesta área. Para estabelecer uma definição uniforme a Acute Dialysis Quality Initiative (ADQI) formulou a classificação: risk, injury, failure, loss and end-stage kidney (RIFLE) ${ }^{22}$. Porém, no presente estudo, como foi utilizado o escore ATN-ISS descrito por Liaño ${ }^{12}$, usamos a definição de IRA descrita no artigo original.

A prevalência de IRA em nosso estudo $(1,05 \%)$ foi semeIhante a outros estudos publicados 2, 4,11, 12, 23, 24,25. A mortalidade geral dos pacientes com IRA também foi semelhante a outros estudos $(48 \%)^{12,26-28}$, e foi maior do que a mortalidade geral dos pacientes internados nas enfermarias do hospital (6\%). Existem poucos estudos epidemiológicos em IRA no Brasil. Castro M C et al ${ }^{29}$ fizeram uma análise retrospectiva de 91 casos de IRA por NTA (não definida) no período de 1980 a 1982, comparando com revisão feita no mesmo serviço no período de 1957 a 1966 , em relação aos fatores etiológicos. Os autores demonstraram que houve uma mudança no padrão etiológico da IRA. Silva LAM et al. ${ }^{23}$ avaliaram de forma retrospectiva 300 prontuários de pacientes que tiveram o diagnóstico de IRA registrado, internados em três hospitais, no período de janeiro de 1982 a março de 1991. Observaram que em 0,17\% das internações foi feito diagnóstico de IRA. A mortalidade total nos pacientes com IRA foi de 39,7\%. Santos WJ at al. ${ }^{30}$, em estudo realizado entre 1997 a 2000, avaliaram pacientes com NTA em UTI e observaram que a etiologia multifatorial foi responsável por $90 \%$ dos casos e apresentou maior mortalidade.

Analisando os dados globais, observamos que $47 \%$ dos pacientes já apresentavam IRA à admissão hospitalar (IRA comunitária) e a maioria destes (65\%) foi tratada fora da UTI. Um maior número de casos de IRA hospitalar foi visto na UTI $(55,5 \%)$, o que está de acordo com a maior prevalência de IRA séptica e de insuficiências orgânicas no grupo UTI, como já observado por outros autores ${ }^{14,31}$.

\section{Fatores prognósticos em IRA}

Em 1998, Liaño et al. ${ }^{14}$, compararam IRA UTI vs não-UTI e observaram que a mortalidade aumentava com a elevação do número de falência de órgãos em ambos os setores, observando também, que os marcadores prognósticos eram os mesmos.

A mortalidade dos pacientes tratados na UTI foi de $85 \%$ e no grupo não-UTI de $18 \%$. Dado semelhante foi observado por D'Ávila et al. que analisaram retrospectivamente 124 pacientes com IRA em UTI, submetidos à diálise com a finalidade de criar um índice prognóstico local, comparando-o com o APACHE II. A mortalidade foi de $81 \%$ e houve bom desempenho do índice local $^{6}$. Lima $\mathrm{EQ}^{32}$, analisando 324 pacientes com IRA, também observaram mortalidade hospitalar de 85\%. Em 2006, Mataloun SE et al. analisaram 221 casos consecutivos de IRA na UTI e concluíram haver uma alta prevalência de IRA, com mortalidade de $76,2 \%{ }^{3}$.

Em nosso estudo, observamos que idade e alteração de variáveis fisiológicas foram mais prevalentes na UTI. Porém, com relação às doenças crônicas, houve um padrão diferente nos dois grupos e, não podemos dizer que patologias crônicas (às quais é atribuído maior número de pontos no escore APACHE) foram mais prevalentes em qualquer um dos dois grupos. Num contexto geral, as características que possuem maior "peso" no escore APACHE II estiveram mais presentes nos pacientes tratados na UTI. Com relação ao escore ATN-ISS, todas as variáveis utilizadas foram mais prevalentes na UTI.

Analisando as características da IRA, vimos que pacientes oligúricos, com necessidade dialítica e menor índice de recuperação renal foram mais prevalentes na UTI e correlacionaram-se com maior mortalidade. Dados semelhantes foram encontrados por Carmo et al, que, analisando 49 pacientes com IRA dialítica internados em UTI, observaram elevada mortalidade $(67,3 \%)$, associada à falência de múltiplos órgãos e oligúria ${ }^{33}$.

O tipo de diálise mais utilizado em pacientes tratados fora da UTI foi HDI e em UTI CVVHD, o que reflete a maior gravidade dos pacientes tratados na UTI, já que procedimentos contínuos foram mais utilizados para pacientes com falência cardiovascular e sépticos. A maior mortalidade dos pacientes que utilizaram procedimentos contínuos reflete provavelmente a gravidade dos mesmos, não tendo correlação direta com o tipo de procedimento dialítico utilizado $(p<0,001 ; \mathrm{IC}=6,43-166,97)^{34,35}$.

\section{Escores de mortalidade em IRA}

$O$ uso de escores de mortalidade geral em pacientes com IRA em UTI é relativamente freqüente, sendo mais utilizado o 
APACHE $\|^{6,36-39}$. Apesar disso, os resultados têm sido conflitantes. Alguns autores ${ }^{37,40-42}$ não evidenciaram bom desempenho com o uso do mesmo, enquanto outros ${ }^{34,36,38,39}$ sugeriram ser este um bom preditor de mortalidade em pacientes com IRA. Outro fator que complica esta análise diz respeito ao momento em que o cálculo do escore foi efetuado por cada um dos autores: dia da admissão na UTI ${ }^{1}$, dia da primeira diálise ${ }^{13}$ e dia da avaliação do nefrologista ${ }^{43}$. De fato, alguns autores que obtiveram um desempenho insatisfatório com o APACHE II, comparado com outros escores, atribuíram o insucesso a um viés tempo dependente e, ao fato deste escore não incluir em suas variáveis características da IRA associadas à menor sobrevida12, 40,42,44-46,

No APACHE III foi incluído a variável oligúria. O escore ATN-ISS tem, no conjunto de suas variáveis, a presença de oligúria como fator correlacionado com pior prognóstico. Como oligúria esteve associada com mortalidade na análise multivariada, é possível que esta variável tenha contribuído para o melhor desempenho do ATN-ISS comparado com o APACHE II.

A utilização do escore APACHE II em pacientes tratados fora da UTI, em enfermarias ou unidades de cuidados intermediários já foi realizada em uma série de estudos ${ }^{16,17,18,47}$; porém, o uso do escore APACHE II em pacientes com IRA tratados fora da UTI foi realizado apenas no estudo de Hakim et al. ${ }^{48}$, que utilizou o escore APACHE II para estratificar os pacientes em um estudo realizado para avaliar o uso de membranas biocompatíveis em pacientes com IRA e necessidade dialítica. Nesse estudo, um grupo de pacientes encontrava-se fora da UTI à época da realização do escore $^{49}$. Parker et al, em $1996^{13}$, utilizaram parte dos dados de Hakim et al. ${ }^{48}$ e analisaram o desempenho do APACHE II no dia da diálise, em pacientes com IRA. Dezesseis por cento dos pacientes estavam fora da UTI quando foi realizado o APACHE II, porém esta população não foi analisada separadamente. Estes autores referem ser o dia da diálise o melhor momento para estratificar risco nesta população, porém não foi realizada comparação do escore em momentos distintos no estudo citado.

O ATN-ISS, por sua vez, foi usado por vários autores $7,14,38,39$, isolado e comparado a escores de mortalidade geral e específica, sempre se revelando um bom preditor. Em nosso estudo, para eliminarmos o viés tempo dependente, realizamos o ATN-ISS e o APACHE II no dia da avaliação do nefrologista para pacientes internados na UTI e fora da UTI. A média dos escores foi maior entre os pacientes tratados na UTI e entre os não sobreviventes, e todas as variáveis do escore ATN-ISS (idade, oligúria, hipotensão, icterícia, coma, assistência respiratória) correlacionaram-se com menor sobrevida (dados não mostrados).

Avaliamos os escores com relação à discriminação e calibração. A importância de fazer distinção entre discriminação (como o modelo discrimina sobreviventes de não sobreviventes) e calibração (como o desfecho predito correlaciona-se com o desfecho observado) depende de como o instrumento preditivo vai ser usado. Schuster, $1992^{50}$, refere que, para pesquisadores ou para avaliação de qualidade (comparação de grupos), a calibração é especialmente importante. Para decisões em pacientes individuais, ambos são importantes. O principal objetivo de pesquisadores que utilizam escores prognósticos é a avaliação de grupos. Para avaliar pacientes individuais, além de haver necessidade de uma calibração o mais exata possível, ao analisar a discriminação há necessidade de determinar um ponto de corte para classificar os pacientes em duas categorias (maior e menor probabilidade da ocorrência de um desfecho) para que decisões possam ser tomadas.

Nos pacientes tratados na UTI, o risco-APACHE II mostrou uma área sob a curva (AUC) de 0,78. Para o grupo não-UTI, a área sob a curva (AUC) foi de 0,66. A comparação das AUC não mostrou diferença estatisticamente significante. Isto demonstra um poder discriminatório semelhante em ambas as populações, 0 que equivale a dizer que a capacidade de distinguir entre sobreviventes e não sobreviventes do APACHE II para estas populações foi semelhante. Em estudos prévios $(1,38)$, o escore APACHE II em pacientes com IRA tratados em UTI foi realizado à época da admissão na UTI, realizado quando da avaliação do nefrologista ${ }^{43}$, à época da diálise ${ }^{13}$ e seriado ${ }^{51}$, observamos que o poder discriminatório deste índice nestes estudos foi semelhante àquele visto em nosso estudo. Não há estudos prévios comparando este escore entre a população com IRA tratada na UTI e fora da UTI.

Avaliando a calibração, o escore APACHE II subestimou a mortalidade no grupo UTI. Para o grupo não-UTI houve uma boa calibração, com boa correlação entre mortalidade predita e observada. A calibração refere-se ao grau de correspondência entre a probabilidade de mortalidade predita e a mortalidade observada, e nos mostra, em outras palavras, quanto o escore tende a subestimar ou superestimar a ocorrência de um desfecho na população estudada. Douma et al., em um estudo avaliando a desempenho de 11 modelos preditores de mortalidade (específicos e inespecíficos), mostrou, na análise de calibração do escore APACHE II que este subestimou a mortalidade ${ }^{38}$. Observamos, em nosso estudo, que a calibração foi melhor no grupo não-UTI e isto se deve, provavelmente, ao fato de a mortalidade ser mais baixa neste grupo.

Analisando-se o ATN-ISS, devemos primeiro ressaltar que, no presente estudo, este escore foi utilizado para pacientes com IRA em geral. A AUC para o grupo UTI foi de 0,94 e, para o grupo não-UTI foi de 0,93 , revelando um excelente poder discriminatório deste índice. Em estudos prévios ${ }^{1,38,39}$, este escore sempre obteve bom desempenho, porém em nosso estudo a AUC foi notadamente maior. É possível que o melhor desempenho deste escore em nosso estudo deva-se ao fato de termos incluído pacientes com IRA em geral, o que implica em aior número de casos que carreiam menor gravidade, tendo poucos fatores de risco para mortalidade e, sendo mais fáceis de predizer o desfecho. Além disso, a grande prevalência de pacientes com necessidade de assistência respiratória (42\%), fator que possui o maior coeficiente no escore ATN-ISS $(0,182)$, seguido da alta prevalência de pacientes em coma (39\%), segundo fator com maior coeficiente $(0,150)$ neste escore. Podemos observar também que não houve diferença entre o poder discriminatório, avaliado pela AUC, entre o grupo UTI e não-UTI.

Com relação à calibração do ATN-ISS, vimos uma boa correlação entre a mortalidade predita e observada para o grupo UTI, como observado por Batista et al. ${ }^{52}$, porém no grupo não-UTI este escore superestimou a mortalidade em todos os quintiles, com melhor desempenho no último quintil de risco, onde o risco de morte é maior. Vemos um comportamento deste escore inverso ao APACHE II, com melhor calibração em pacientes que se encontram com maior risco de morte. Isto poderia ser devido ao fato de que nem todas as variáveis do escore APACHE II se correlacionaram com menor sobrevida, fazendo com que este 
escore subestime a mortalidade em nosso estudo. Como todas as variáveis do escore ATN-ISS correlacionaram-se com menor sobrevida, sua mortalidade predita foi semelhante à observada, porém com maior tendência a superestimar a mortalidade.

\section{Conclusão}

As diferenças entre os pacientes com IRA tratados em UTI e fora da UTI indicam maior gravidade dos pacientes tratados em UTI. O APACHE II apresentou discriminação semelhante entre os grupos UTI e não-UTI. O APACHE II subestimou a mortalidade no grupo UTI, porém apresentou boa calibração para o grupo não-UTI. O ATN-ISS apresentou boa discriminação nos grupos UTI e não-UTI, boa calibração no grupo UTI e superestimou a mortalidade no grupo não-UTI. O ATN-ISS apresentou melhor capacidade de discriminação do que o APACHE II (colhido na mesma data) nos grupos UTI e não-UTI. O APACHE II e o ATN-ISS podem ser utilizados para a estratificação de risco de pacientes com IRA tratados fora da UTI em nosso meio.

\section{Conflito de interesse: não há}

\section{SUMMARY}

APACHE II AND ATN-ISS IN ACUTE RENAL FAILURE (ARF) IN INTENSIVE CARE UNIT (ICU) AND NON-ICU

INTRODUCTION. Acute renal failure (ARF) remains highly prevalent with a high rate of morbidity and mortality.

OBJECTIVE. Of this study was to compare use of the APACHE II scoring prognosis with that of the ATN-ISS to determine whether the APACHE I/ could be used for patients with ARF outside the ICU.

Methods. For this purpose,, 205 patients with ARF were accompanied in a prospective cohort. Demographic data, preexisting conditions, organ failure and characteristics of ARF were analyzed. The prognostic scores were performed with the assessment of a nephrologist.

RESULTS. The mean age was $52+18$ years, $50 \%$ were male, $69 \%$ were white, $45 \%$ were treated in ICU and $55 \%$ in other units. Mortality in the ICU group was $85 \%$ and in the non-ICU group $18 \%$. Factors that correlated with higher mortality were more prevalent in the ICU group: age, male, hospitalization with ARF, organ failure, sepsis, septic IRA, oliguria and need of dialysis. Overall, the prognostic markers were the same for both the ICU and non-ICU groups. The discrimination with the APACHE II was similar in both, ICU and non-ICU groups and calibration was better in the non-ICU group. The ATN-ISS achieved good discrimination in both the ICU and non-ICU groups, but, regarding calibration, there was a discreet overestimating of mortality in the non-ICU group. The ATN-ISS showed a greater capacity for discrimination than the APACHE /I in both the ICU and non-ICU groups.

CONCLUSION. It was concluded that the APACHE // and ATN-ISS scores could be used for stratification of risk in patients with ARF treated outside of the ICU in Brazil. [Rev Assoc Med Bras 2009; 55(4): 434-41]

KEY WORDS: APACHE II. ATN-ISS. Acute Renal Failure. Intensive Care Unit.

\section{REFERÊNCIAS}

1. Liaño F. Severity of acute renal failure: the need of measurement. Nephrol Dial Transplant. 1994;9(4 Suppl):229-238.

2. Elasy TA, Anderson RJ. Changing demography of acute renal failure. Semin Dial. 1996;9:438-43.

3. Matoloun SE, Machado FR, Sena AR, Guimarães HP, Amaral JG. Incidence, risk factors and prognostic factors of acute renal failure in patients admitted to an intensive care unit. Braz J Med Biol Res. 2006;39:1339-47.

4. Kaufman J, Dhakal M, Patel B, Hamburger R. Community-acquired acute renal failure. Am J Kidney Dis. 1991;17:191-8.

5. Schuster DP. Predicting outcome after ICU admission: the art and science of assessing risk. Chest. 1992;102:1861-70.

6. D’Ávila DO, Traezel M, Glock L. Insuficiência renal aguda tratada por diálise em unidade de tratamento intensivo (análise de 124 pacientes consecutivos). J Bras Nefrol. 1997;19:21-31.

7. Balbi AL, Gabriel DP, Barsante RC, Caramori JT, Martin LC, Barreti, P. Mortalidade e prognóstico específico em pacientes com insuficiência renal aguda. Rev Assoc Méd Brás. 2005;51:318-22.

8. Knaus WA, Draper EA, Wagner DP, Zimmerman JE. APACHE II: A severity of disease classification system. Crit Care Med. 1985;13:818-29.

9. Knaus WA, Wagner DP, Draper EA, Zimmerman JE, Nergner M, Bastos PG, et al. The APACHE III Prognostic System. Risk prediction of hospital mortality for critically ill hospitalizad adults. Chest. 1991;100:1619-36.

10. www.cerner.com, acessado em 15 de outubro de 2008

11. Liaño F, Garcia-Martin F, Gallego A, Orte L, Teruel JL, Marcén R, et al. Easy and early prognosis in acute tubular necrosis: a forward analysis of 228 cases. Nephron. 1989;51:307-13.

12. Liaño F, Gallego A, Pascual J, Garcia-Martin F, Teruel JL, Marcen R, et al. Prognosis of acute tubular necrosis: na extended Pospective-ly contrasted study. Nephron. 1993;63:21-31.

13. Parker RA, Himmelfarb J, Tolkoff-Rubin N, Chandran P, Wingard R, Hakim RM. Prognostic of patients with acute renal failure requiring dialysis: results of a multicenter study. Am J Kidney Dis. 1998;32:432-43.

14. Liaño F, Junco E, Pascual J, Madero R, Verde E; The Madrid Acute Renal Failure Study Group. The spectrum of acute renal failure in the intensive care unit compared with that seen in other settings. Kidney Int. 1998;53 (66 Suppl):16-24.

15. Fiaccadori E, Lombardi M, Leonardi S, Rotelli CF, Tortorella G, Borgethi A. Prevalence and clinical outcome associated with preexisting malnutrition in acute renal failure: a prospective cohort study. J Am Soc Nephrol. 1999;10:581-93.

16. Zimmerman JE, Wagner DP, Knaus WA, Williams JF, Kolakowski D, Draper EA. The use of risk predictions to identify candidates for intermediate care units. Implications for intensive care utilization and cost. Chest. 1995;108:490-9.

17. Lundberg JS, Perl TM, Wiblin T, Costigan MD, Dawson J, Nettleman MD, et al. Septic shock: an analysis of outcomes for patients with onset on hospital wards versus intensive care units. Crit Care Med.1998; 26:1020-4.

18. Goldhilldr, Worthingtin L, Mulcahy A, Tarling M, Sumner A. The patients-atrisk team: identifying and managing seriously il ward patients. Anaesthesia. 1999;54:853-60.

19. Knaus WA, Draper EA, Wagner DP, Zimmerman JE. Prognosis in acute organsystem failure. Ann Surg. 1985;202:685-93.

20. Hanley JÁ, Mcneil BJ. The meaning and use of the area under a receiver operating characteristic (ROC) curve. Radiology. 1982;143:29-36.

21. Delong ER, Delong DM, Clarke-Pearson DL. Comparing the areas under two or more correlated receiver operating characteristic curves: a nonparametric approach. Biometrics. 1988;44:837-45.

22. Bellomo R, Ronco C, Kellum JA, Mehta RL, Palevsky P, The ADQI workgroup. Acute renal failure - definition, outcome measures, animal models, fluid therapy and information technology needs: the Second International Consensus Conference of the Acute Dialysis Quality Initiative (ADQI) Group. Crit Care.2004;8:R204-12

23. Silva LM, Cauduro CC, Mezzomo NF, Pansard HM, Arantes LC, Rempel W, et al. Insuficiência Renal Aguda: análise retrospectiva de 300 casos. J Bras Nefrol. 1991;13:105-14.

24. Fesst TG, Round A, Hamad S. Incidence of severe acute renal failure in adults: results of a community based study. BMJ. 1993;306:481-3.

25. Liaño F, Pascual J; The Madrid Acute Renal Failure Study Group. Epidemiology of acute renal failure: a prospective, multicenter, community-based study. Kidney Int. 1996;50:811-8.

26. Turney $\mathrm{JH}$. Why is mortality persistently high in acute renal failure? Lancet. 1990;335:971

27. Turney JH, Marshall DH, Browngon AM, Ellis CM, Parsons FM. The evolution of acute renal failure 1956-1988. Q J Med. 1990;74:83-104.

28. Biesenbach G, Zazgornik J, Kiser W, Grafinger P, Stuby U, Necek S. Improvement in prognosis of patients with acute renal failure over a period of 15 years: an analysis of 710 cases in a dialysis center. Am J Nephrol. 1992;12:319-25. 
29. Castro MM, Martins V, Nadalin F, Malheiros P, Sabbaga E, Marcondes M. Mudança do perfil etiológico da insuficiência renal aguda. J Bras Nefrol.1983;5:26-8.

30. Santos WJ, Zanetta DM, Pires AC, Lobo SM, Lima EQ, Burdmann EA. Patients with ischaemic, mixed and nephrotoxic acute tubular necrosis in the intensive care unit - a homogeneous population? Crit Care. 2006;10:R68.

31. Neveu H, Kleinknecht D, Brivet F, Loirat P, Landais P; The French Study Group on Acute Renal Failure. Nephrol Dial Transplant. 1996;11:293-299.

32. Lima EQ, Dirce MT, Castro I, Yu L. Mortality risk factors and validation of severity scoring systems in critically ill patients with acute renal failure. Ren Fail. 2005;27:547-56.

33. Carmo PV, Amaral CF, Paiva AB, Ribeiro CS, Ramalho GT, Bastos MG, et al. Insuficiência Renal Aguda Dialítica: Experiência em Hospital Universitário. J Bras Nefrol. 2006;28:7-14.

34. Bellomo R, Boyce N. Acute continuous hemodiafiltration: a prospective study of 110 patients and a review of the literature. Am J Kidney Dis. 1993;21:508-18.

35. Mehta R. Modalities of dialysis for acute renal failure. Semin Dial. 1996;9:469-75.

36. Maher ER, Robinson KN, Scoble JE, Farrimond JG, Browne DRG, Sweny P, et al. Prognosis of critically-ill patients with acute renal failure: APACHE II score and other predictive factors. Q J Med. 1989;72:857-66.

37. Chertow GM, Christiansen CL, Cleary PD, Munro C, Lazarus JM. Prognostic estratification in criticaly ill patients with acute renal failure requiring dialysis. Arch Intern Med. 1995;155:1505-11.

38. Douma CE, Redekop WK, Van Der Meulen JH, Van Holden RW, Haeck J, et al. Predicting mortality in intensive care patients with acute renal failure treated with dialysis. J Am Soc Nephrol. 1996;8:111-7.

39. Batista PB. Prognóstico de pacientes com insuficiência renal aguda [dissertação]. São Paulo: Escola Paulista de Medicina, Universidade Federal de São Paulo; 1998.

40. Schaefer JH, Jochimsen, Keller F, Wegscheider K, Distler A. Outcome prediction of acute renal failure in medical intensive care. Intensive Care Med. $1991 ; 17: 19-24$

41. Fery-Lemonnier E, Landais $P$, Loirat $P$, Kleinknecht $D$, Brivet $F$. Evaluation of severity scoring systems in ICU- translation, conversion and definition ambiguities as a source of inter-observer variability in APACHE II, SAPS and OSF. Intensive Care Med. 1995;21:356-60.
42. Barton IK, Hilton PJ, Taub NA, Warburton FG, Swan AV, Dwight J, et al. Acute renal failure treated by hemofiltration: factors affecting outcome. Q J Med. 1993;86:81-90.

43. Cosentino F, Chaff C, Piedmonte M. Risk factors influensing survival and ICU acute renal failure. Nephrol Dial Transplant.1994;9 (4):179-82.

44. Cioffi WG, Ashikaga T, Gamelli RL. Probability of surviving postoperative acute renal failure. Development of a prognostic index. Ann Surg.1984; 200(2):205-11.

45. Bullock ML, Umen AJ, Finkelstein M, Keane WF. The assesment of risk factors in 462 patients with acute renal failure. Am J Kidney Dis. 1985;5(2):97-103.

46. Lohr JW, McFarlane MJ, Grantham JJ. A clinical index to predict survival in acute renal failure patients requiring dialysis. Am J Kidney Dis. 1988;11(3):254-9.

47. Tumbarello $M$, Taconelli $E$, Donati KG, Leone $F$, Morace $G$, Cauda $R$, et al. Nosocomial bloodstream infections in HIV-infected patients: attributable mortality and extension of hospital stay. J Acquir Immune Defic Syndr Hum Retrovirol. 1998;19(5):490-7.

48. Hakim RM, Wingard RL, Parker RA. Effect of the dialysis membrane in the treatment of patients with acute renal failure. New England $\mathrm{J}$ Medicine. 1994;331(20):1338-42.

49. Kaplan AA, Paganini EP, Bosch JP. Effects of the dialysis membrane in acute renal failure. New Engl J Med. 1995;332(14):961-2.

50. Schuster DP. Predicting outcome after ICU admission: the art and science of assessing risk. Chest. 1992;102(6):1861-70.

51. Mehta R, Farkas A, Pascual M, Fowler W. Effect of serial APACHE and organ failure scores on prediction of mortality in acute renal failure. J Am Soc Nephrol. 1995;6:550.

52. Batista PB, Cendoroglo Neto M, Santos OF, Bacelar AC, Campos GB, Santos ES. Evaluation of prognostic indexes in critical acute renal failure patients. Ren Fail. 2004;26(5):545-52.

Artigo recebido: 06/08/08 Aceito para publicação: 28/01/09 